already been used to identify proteins that are affected in tumors and hence lead to specific targets for drugs to treat different forms of cancer. In a similar approach, 2D electrophoresis is being carried out using postmortem central nervous system (CNS) from a large cohort of subjects with schizophrenia and tissue from the same CNS region of subjects with no history of psychiatric or neurological disease. We have modified the approach to 2D electrophoresis so that the analyses will measure the levels of over 10000 proteins in each tissue sample. In collaboration with the Centre for Mathematics and Statistics of Complex Systems, we are now analyzing our 2D gels to identify grouping of differential protein expression patterns that might define different illnesses within the syndrome of schizophrenia. In this presentation, a proof of principal study to differentiating schizophrenia and bipolar disorder will be presented.

$08-03$

\section{From biological marker to endophenotype: the role of animal models}

\author{
D Eyles ${ }^{1,2}$, T Burne' ${ }^{2}$ J McGrath ${ }^{2,3}$ \\ 'School of Biomedical Sciences, University of Queensland; ${ }^{2}$ Queensland Centre for \\ Mental Health Research, The Park, Wacol, Queensland, Australia; and \\ ${ }^{3}$ Department of Psychiatry, University of Queensland, Brisbane, Australia
}

Our group has been exploring the concept that developmental vitamin D (DVD) deficiency may be the plausible neurobiological explanation for several important epidemiological correlates of schizophrenia, namely 1) the excess winter/spring birth rate, 2) increased incidence of the disease in second-generation Afro-Caribbean migrants and 3) increased urban birth rate. We have produced two pieces of direct support for this hypothesis in patients. DVD deficiency therefore is a plausible 'biological marker' for schizophrenia. We have recently resolved a major technological barrier that will now allow us to test this hypothesis in a major European developmental biobank. The genetic factors or heritability indicators that would endow DVD deficiency as a valid schizophrenia endophenotype are emerging. Our group has established a highly informative animal model to study the effects of DVD deficiency on brain development. This animal model reproduces the gross pathological features of the disease, that is, ventriculomegaly as well as sensitivity to amphetamine- and MK-801-induced hyperlocomotion and impairments in latent inhibition, behaviours analogous to the positive and negative symptoms of the disease in patients. We are now examining whether this model internally fulfils certain molecular endophenotypic criteria. Our initial data are encouraging as we find a $36 \pm 11 \%$ decrease in the developmental expression of the candidate endophenotypic gene for schizophrenia, COMT. This animal model continues to provide good face and some construct validity with the disease in patients and illustrates how animal models can be used to progress plausible biological markers for schizophrenia into endophenotypes.

08-04

\section{Endophenotypic biobehavioural markers for schizophrenia: how close are we to finding the Holy Grail? \\ P Ward ${ }^{1,2}$ \\ 'University of New South Wales, Sydney, New South Wales, Australia; and ${ }^{2}$ Schizophrenia Research Unit, Sydney South West Area Health Service, Liverpool, Australia}

Research over several decades has showed that current operational diagnoses are unlikely to map directly onto the biological substrates of schizophrenia. This has led to increased interest in identifying endophenotypic markers that may provide an intermediate link to underlying genes. This talk will review some of the more promising biobehavioural markers currently being investigated, such as P50 sensory gating, reduced mismatch negativity amplitude and regionally specific gray matter volume loss, and highlight some of the challenges that still remain to be overcome. These include the potential impact of various potential confounds (eg treatment effects and comorbidity). Our current state of knowledge highlights the need for large-scale collaborative efforts that could provide convincing evidence for the utility of putative biobehavioural markers in furthering our understanding of the etiology of schizophrenia.

\section{8-05}

\section{'Kraepelinian' and 'Bleulerian' schizophrenia: a genetic dissection of a cognitive endophenotype}

\section{A Jablensky $y^{1,2}, \mathrm{~J}$ Hallmayer ${ }^{1,3}, \mathrm{M}$ Dragovic ${ }^{2}$, J Badcock ${ }^{1,2}$, L Kalaydjieva ${ }^{4}$}

${ }^{1}$ UWA School of Psychiatry and Clinical Neuroscience; ${ }^{2}$ Centre for Clinical Research in Neuropsychiatry; ${ }^{3}$ Stanford University School of Medicine Department of Psychiatry and Behavioural Sciences; and ${ }^{4}$ Western Australian Institute of Medical Research, Perth, Australia 
Cognitive deficit was conceived by Emil Kraepelin (1919) to be a core feature of a single disease, dementia praecox. In contrast, Eugen Bleuler (1923) hypothesized that schizophrenia was the 'common final pathway' for an etiologically mixed group of disorders. The controversy engendered by these two great clinicians survives unscathed in the present diagnostic concepts of schizophrenia. Because schizophrenia is clinically heterogeneous and genetically complex, ICD-10 and DSM-IV diagnoses may not provide the optimal phenotypes for genetic analysis. We developed a novel, endophenotype-based approach to the search for susceptibility genes by generating a composite phenotype that integrates multiple measurements of neurocognition, neurological signs and personality traits using a variant of latent class analysis known as grade of membership. The WA Family Study of Schizophrenia sample (112 families, 388 individuals) yielded two distinct neurocognitive phenotypes, each correlated with schizophrenia: one indexing pervasive neurocognitive deficit and predominantly 'negative' symptoms, and one neurocognitively unimpaired, with florid 'positive' psychotic symptoms. The quantitative neurocognitive trait was used in linkage analysis as a liability covariate, on which each individual in the sample (affected and unaffected) was scored. A 10-cM genome scan, followed by ordered sets analysis resulted in a robust linkage signal (lod score $>3.4$ ) for the neurocognitive deficit subtype on chromosome 6p2522 , whereas the neurocognitively unimpaired subtype was definitively excluded for linkage to the same region. This work supports 'splitting' schizophrenia into genetically distinct subtypes and shows an increase in power resulting from use of composite quantitative endophenotypes.

\section{The Role of Apolipoprotein E in Neuropsychiatric Disorders}

\section{S Sundram}

Mental Health Research Institute, Parkville, Victoria, Australia

\section{Overview}

The most robust genetic predictor of sporadic Alzheimer's disease (AD) is the $\varepsilon 4$ allele of the apolipoprotein E (apoE) gene. This has spawned much interest into the relevance of this lipidbinding protein in this and other neuropsychiatric disorders and the roles it may play in the central nervous system (CNS). This symposium will present work examining the role of the apoE protein in neuronal function and CNS disorders focusing upon $\mathrm{AD}$, schizophrenia and bipolar disorder. Novel data exploring both central and peripheral functions for apoE and its dysregulation will highlight the relevance of this protein in the pathology of these disorders.

\section{9-01}

\section{Role of apolipoprotein E in Alzheimer's disease}

\section{RN Martins}

Centre of Excellence for Alzheimer's Disease Research and Care, Edith Cowan University, Perth, Western Australia, Australia

The $\varepsilon 4$ allele of apolipoprotein E (APOE) is currently the major genetic risk factor for Alzheimer's disease (AD) and is associated with $25 \%-50 \%$ of late-onset AD. Several studies, including our own with the Australian Caucasian population, show that the AD risk associated with the APOE genotype is exerted across most populations worldwide with the exception of the Hispanic and African American populations. However, while the $\varepsilon 4$ allele is generally recognized to play an important role in the pathogenesis of $\mathrm{AD}$, its mechanism of action remains controversial and is poorly understood. Others and we have shown in vitro that one potentially important mechanism of action involves its role in the metabolism of beta amyloid $(A \beta)$ thought to be central to the pathogenesis of $\mathrm{AD}$. We showed the ApoE $\varepsilon 4$ isoform is deficient in binding to $A \beta$ and clearing it from the extracellular space, an event that appears to be through binding of ApoE/A $\beta$ complexes to LRP. Recently, we have shown in vivo that mouse apoE retards clearance from peripheral tissues such as liver and kidney of $A \beta$ injected into the circulation. Furthermore, we show that $A \beta$ administered peripherally does not result in uptake by the brain which is consistent with previous transgenic work for human $A \beta$ showing that peripheral $A \beta$ does not directly contribute to the pool of brain $A \beta$. Taken together with recent findings that proteins that bind $A \beta$ in the periphery enhance its clearance from the brain together with recent findings that proteins that bind $A \beta$ in periphery enhance its clearance from the brain, we propose that human apoE alters $A \beta$ clearance in an isoform-specific manner in vivo such that apoE $\varepsilon 2$ is most efficient and apoE $\varepsilon 4$ is least efficient. 